EDITORIAL

\title{
The changing face of acute myocardial infarction
}

\author{
N Danchin
}

Heart 2005;91:705-706. doi: 10.1136/hrt.2004.052399

\section{The appointment of new cardiologists and their involvement in emergency care may lead to decreased mortality in patients suffering acute myocardial infarction}

O ver the past 15 years, clinical researchers have documented the efficacy of both new (or older) medications and percutaneous interventions in patients with acute myocardial infarction. An integrated approach, mixing the use of pharmacologic agents and interventional procedures, has been promoted, with the hope of reducing early (and late) mortality. However, translating recommendations into the "real world" of everyday clinical practice is a difficult exercise and obviously has more chances of success when the educational efforts are, at least initially, directed to a more limited number of physicians, namely, specialists in the target field.

In this regard, the paper by Murphy and colleagues $^{1}$ provides interesting data by investigating the evolution of early mortality of acute myocardial infarction, 10 years apart (from the late 1980s to the late 1990s), in Scotland, and this in relation to the appointment of new cardiologists involved in emergency care. All acute hospitals in Scotland participated in the study. Overall, median 30 day mortality decreased by an absolute $2.4 \%$ (or a $12 \%$ relative decrease), a difference highly significant after multivariate adjustment for potential confounders. Importantly, between-hospital variations decreased considerably from the first to the second period (difference between highest and lowest survival: $13.0 \%$ in period $1 \vee 7.6 \%$ in period 2). Likewise, nine of 26 hospitals in the 1988-1991 period but only two in the 1998-2001 period had an adjusted risk of death significantly higher than average. These results therefore document the improved, but also more homogeneous, results achieved in the management of patients with acute myocardial infarction in Scotland over a relatively short period of time. As, from period 1 to period 2, at least one cardiologist has been appointed in each Scottish hospital, the authors suggest that both the decrease in mortality and the lower betweenhospital variation may be related to the appointment of new cardiologists and their involvement in emergency care.

Correspondence to: Professor Nicolas Danchin, Hôpital Européen Georges Pompidou, Paris, Service de Cardiologie, HEGP, 20 rue Leblanc, 75015 Paris, France; nicolas.danchin@ egp.ap-hop-paris.fr APPOINTMENT OF NEW CARDIOLOGISTS Indeed this suggestion seems logical: the appointment of new cardiologists is likely to result in both improved and more rapid diagnosis tion, and also a more appropriate application of guidelines and recommendations. In a recent survey that we carried out in a large sample of French general practitioners, consultation with a cardiologist in the preceding year was an independent determinant of more adequate implementation of guidelines and better risk factor control in patients with chronic coronary artery disease or at high cardiovascular risk (personal data on file).

However, the data provided in the study cannot demonstrate that the appointment of more cardiologists is the sole explanation for improved outcomes: once myocardial infarction has been recognised, what becomes crucial is the immediate use of appropriate treatments. In other words, appointing a cardiologist specialised in emergency care will have a positive impact only as long as the cardiologist will be reasonably competent and will conform to the most recent recommendations.

In this regard, the reader would definitely have liked to know what changes were observed in the actual management of the patients from the late 1980 s to the late 1990s and early 2000s. During this period of time, emphasis has been put both on the importance of reperfusion therapy and on the use of concomitant medications such as angiotensin converting enzyme (ACE) inhibitors. As regards reperfusion therapy, the result of ISIS-2 (second international study of infarct survival), demonstrating the synergistic action of aspirin and thrombolysis, were published in 1998. ${ }^{2}$ It is therefore unlikely that the recommendations derived from this trial would have been widely applied before 1990 (that is, during the first half of the reference period). In addition, direct angioplasty was not used during the whole duration of the first period. The studies documenting the superiority of primary angioplasty over intravenous thrombolysis, and the major role of time delay on the efficacy of thrombolysis, were published much later. ${ }^{4}$ Similarly, the positive role of early ACE inhibition was documented after the end of the reference period. ${ }^{5}$ Consequently, major changes in the management of acute myocardial infarction are likely to have occurred from period 1 to period 2, and this not only because new cardiologists were appointed, but above all because medical knowledge progressed during that time. In the French experience, important changes in the early management of patients with acute myocardial infarction have been observed between 1995 and of cardiac conditions such as myocardial infarc-
2000, and this in spite of the fact that similar numbers of cardiologists were involved in acute cardiac care between the two surveys. ${ }^{5}{ }^{6}$ These changes resulted in a significant decrease in early mortality. ${ }^{7}$ 


\section{TIME DELAY FROM SYMPTOM ONSET}

Finally, another major aspect of the management of myocardial infarction is the time delay from symptom onset to delivery of appropriate care, particularly reperfusion therapy. Here again, one would have liked to know whether time to hospital admission (and/or time to first medical contact) had varied in Scotland between the two study periods. In this respect, the French experience is particularly disappointing with, if anything, longer time delays in 2000 than in $1995 .^{8}$

Overall improvement in early outcomes certainly has several explanations; improved scientific knowledge leading to more appropriate medical care is central. As suggested by Murphy and colleagues, ${ }^{1}$ implementation of guidelines by more specialised medical personnel is also important. In the specific case of acute myocardial infarction, however, where every single minute matters, one cannot hope for much further improvement in outcomes in the absence of adequate, public oriented information in order to shorten the duration of acute myocardial ischaemia. And this may well be the most difficult task cardiologists will be confronted with in the next decade.

\section{REFERENCES}

1 Murphy NF, Maclntyre K, Stewart S, et al. Reduced between-hospital variation in short term survival after acute myocardial infarction: the result of improved cardiac care? Heart 2005;91:726-30.

2 ISIS-2 Collaborative Group. Randomised trial of intravenous streptokinase, oral aspirin, both, or neither among 17187 cases of suspected acute myocardial infarction: ISIS-2, Lancet 1988;ii:349-60.

3 Weaver WD, Simes RJ, Betriu A, et al. Comparison of primary coronary angioplasty and intravenous thrombolytic therapy for acute myocardial infarction: a quantitative review. JAMA 1997;278:2093-8.

4 Boersma E, Maas ACP, Deckers JW, et al. Early thrombolytic treatment in acute myocardial infarction: reappraisal of the golden hour. Lancet 1996;348:771-5.

5 Domanski MJ, Exner DV, Borkowf $\mathrm{CB}$, et al. Effect of angiotensin converting enzyme inhibition on sudden cardiac death in patients following acute myocardial infarction. A meta-analysis of randomized clinical trials. J Am Coll Cardiol 1999;33:598-604

6 Danchin N, Vaur L, Genès N, et al. Management of acute myocardial infarction in intensive care units in 1995: a nationwide French survey of practice and early hospital results. J Am Coll Cardiol 1997;30:1598-605.

7 Hanania G, Cambou JP, Guéret P, et al for the USIC 2000 Investigators. Management and in-hospital outcome of patients with acute myocardial infarction admitted to intensive care units at the turn of the century: results from the French nationwide USIC 2000 registry. Heart 2004;90:1404-10.

8 Danchin N, Ghobrial H, Hanania G, et al. Changes in in-hospital management and outcome of acute myocardial infarction in the late 1990s: the nation-wide French 1995 and 2000 registries. Eur Heart J 2002;23(abstract suppl):625

\section{IMAGES IN CARDIOLOGY}

\section{A guide catheter removed a massive intracoronary thrombus: a case of acute coronary syndrome}

A 68 year old man without previous history of cardiac disease was admitted with syncope. The ECG showed ST segment elevation in leads II, III, and aVF, as well as advanced atrioventricular block. The right coronary artery angiogram showed a luminal filling defect suggestive of thrombus just proximal to an occlusion (panel A). It was decided to perform percutaneous coronary intervention for this lesion, using of a distal protection device and aspiration catheter. Monitoring of the blood pressure at the tip of a 7 French sized guide catheter suddenly became impossible after an aspiration catheter was pulled out. The guide catheter was found to be displaced from the ostium of the right coronary artery. We assumed that the occlusion had occurred at the tip of the guide catheter, therefore we tried to remove the whole system from the body. Fortunately, it was pulled out with negative pressure on the entire guide catheter and a massive thrombus was also aspirated (panels B and C). After that, we reinserted the catheter and performed coronary intervention. Finally, a stent was implanted at the culprit stenosis at the middle of the right coronary artery. We confirmed that TIMI III coronary

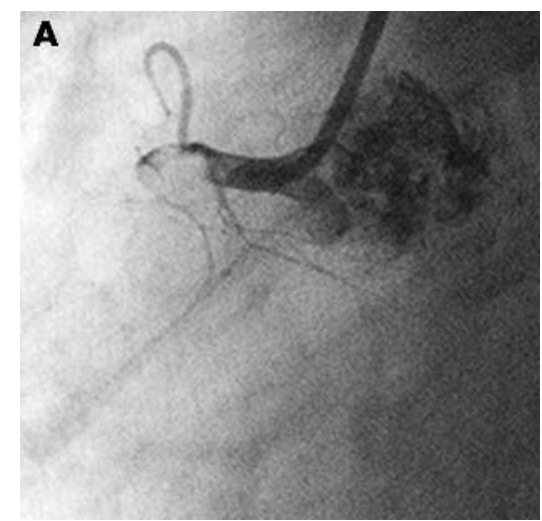

(A) Right coronary artery angiogram showing a luminal filling defect suggestive of thrombus just proximal to an occlusion.

blood flow was restored without residual intracoronary thrombi.

Because the aspiration catheter was pulled out through the guide catheter under negative pressure, the guide catheter itself may have acted as an effective aspiration system.

T Ono, H Hara, M Nakamura harahisa@dl.dion.ne.jp
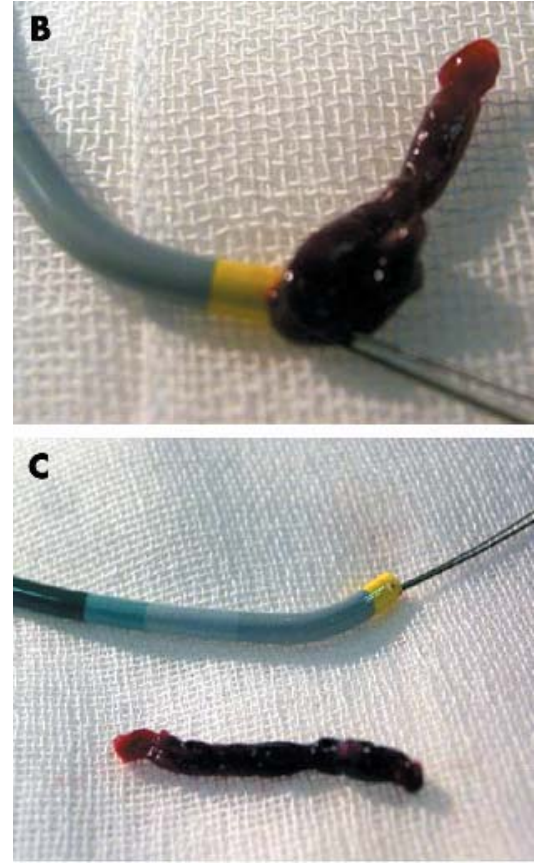

(B) The guide catheter removed from the body. Thrombus is wedged at the tip of the guide catheter. (C) A massive thrombus related acute coronary syndrome was resolved by this fortuitous aspiration system. 